\title{
The role of hysterectomy in the treatment of gestational trophoblastic neoplasms: a single-center experience
}

Nur Gozde Kulhan, Mehmet Kulhan, Umit Arslan Nayki, Cenk Nayki, Nahit Ata, Pasa Ulug, Hulya Toklucu

Gynecology and Obstetrics Department, Erzincan University, Erzincan, Turkey

Submitted: 23 January 2017

Accepted: 6 March 2017

Arch Med Sci Civil Dis 2017; 2: e37-e40

DOI: https://doi.org/10.5114/amscd.2017.66527

Copyright (c) 2017 Termedia \& Banach

\section{Abstract}

Introduction: The main treatment method of gestational trophoblastic neoplasms (GTNs) is chemotherapy. In some situations, surgical treatment including hysterectomy and metastasectomy is required. This study aims to evaluate the experience of a single center with the use of hysterectomy in the treatment of GTNs.

Material and methods: Between May 1995 and March 2014, a total of 35 patients treated for GTNs in our center were retrospectively evaluated. Fourteen of them had undergone a hysterectomy as part of their treatment. Chemotherapy regimens prior to hysterectomy, operation indications, post-hysterectomy treatments, morbidity and mortality rates, follow-up and prognosis were evaluated. Descriptive analyses were used to present the findings.

Results: Mean age at the time of the hysterectomy was $41 \pm 5.7$ (range: $18-58$ ). Ten $(71.4 \%)$ patients received single-agent or multi-agent chemotherapy prior to the hysterectomy. Hysterectomy indications were resistance to chemotherapy in $7(50 \%)$ cases, patient's preference in $4(28.5 \%)$ cases, and intractable bleeding in $3(21.5 \%)$ cases. All hysterectomies were performed transabdominally. There was no mortality or major operative morbidity. Five patients maintained their chemotherapy after hysterectomy as well because they had pulmonary metastasis. Four patients did not receive chemotherapy either preoperatively or postoperatively. Mean follow-up duration after the hysterectomy was $92.6 \pm 38.5$ months (range: $39-180$ months). There was no disease recurrence or death during this period.

Conclusions: Hysterectomy is a safe and effective treatment method, especially for GTNs which are resistant to conventional treatment.

Key words: adjuvant hysterectomy; gestational trophoblastic neoplasia, surgical treatment.

\section{Introduction}

The term gestational trophoblastic neoplasia (GTN) is used when molar and nonmolar pregnancies become malignant. The GTN comprises specific histologic entities, including invasive mole, choriocarcinoma, placental site trophoblastic tumor (PSTT) and epithelioid trophoblastic tumor (ETT). Prior to the development of effective chemotherapy for GTN, the majority of patients with disease located in the uterus were cured

\author{
Corresponding author: \\ Mehmet Kulhan \\ Gynecology and Obstetrics \\ Department \\ School of Medicine \\ Erzincan University \\ Basbaglar Mah \\ 24030 Erzincan, Turkey \\ Phone: +90 5054918090 \\ E-mail: Mehmet_kulhan@ \\ yahoo.com
}


with hysterectomy, whereas metastatic disease was almost uniformly fatal [1]. With the use of sensitive quantitative assays for human chorionic gonadotropin ( $\mathrm{hCG}$ ) and highly effective chemotherapy, most women with GTN can be cured and their reproductive function preserved, provided that they are managed according to well-established guidelines [2]. However, women are at risk for both resistant and recurrent GTN, which will require further therapy. Even then, women who experience a recurrence have a good chance of achieving remission.

Gestational trophoblastic neoplasia is basically treated by chemotherapy. However, decisions regarding surgery must be individualized based on the clinical scenario. The efficacy, feasibility, and type of surgery vary depending on the site and extent of metastases. Very few patients need a hysterectomy [3, 4]. Although surgery has assumed a lesser role in the management of women with malignant gestational trophoblastic disease, selected surgical procedures remain important for treatment [5]. Surgical intervention may be required for chemoresistant disease in the uterus or at metastatic sites, and for life-threatening complications such as hemorrhage, bowel or urinary obstruction, or infection [6-8]. For patients undergoing a hysterectomy, it is important to note that the removal of the ovaries is usually not indicated, even in the presence of theca lutein cysts. Symptomatic ovarian theca lutein cysts can be aspirated if indicated, but otherwise will regress spontaneously after treatment.

The objective of this study was to evaluate the role of adjuvant hysterectomy procedures in the management of GTN.

\section{Material and methods}

Between 1995 and 2014, 35 patients diagnosed with GTN were treated with chemotherapy at our clinics. Patients were grouped according to the International Federation of Gynecology and Obstetrics (FIGO) stage and risk score. A FIGO risk score of $\geq 7$ or the presence of one or more risk factors according to the clinical classification system was indicative of high-risk disease. Of the 35 patients, 21 (60\%) were assigned to the nonmetastatic group, and 14 (40\%) to the metastatic group. In the metastatic group, 7 patients were in the low-risk group and 7 were in the high-risk group. From this patient population, we identified 14 patients who underwent hysterectomy procedures as part of GTN treatment. Chemoresistant disease was defined as a plateau or increase in the level of hCG when the patient had achieved either a partial response (>50\% decrease in hCG titers compared with the value at therapy initiation) or no response $(\leq 50 \%$ decrease in hCG ti- ters compared with the value at therapy initiation) Chemotherapy regimens prior to hysterectomy, indications of hysterectomy, morbidity and mortality, and post-hysterectomy treatment, follow-up, and prognosis were evaluated. Descriptive analyses were used to evaluate the findings.

\section{Results}

The median age of the patients at presentation was 41 years (range: 18-58 years). Forty percent (14/35) of these patients had a hysterectomy. Ten $(71.7 \%)$ patients received single-agent or multi-agent chemotherapy prior to hysterectomy. Seven patients had a pretreatment hCG level of $\geq 40,000 \mathrm{mlU} / \mathrm{ml}$. Seven patients had an interval of $\geq 4$ months from the antecedent pregnancy to treatment; FIGO stage was I in 20, II in 1, III in 7 , and IV in 7 patients. In the metastatic group, 7 women were in the low-risk group and 7 were in the high-risk group. Pathology reports of surgical specimens identified 9 cases of choriocarcinoma, 3 cases of invasive mole and 2 cases of placental site tumor.

In 14 patients, hysterectomy procedures were performed. The presurgery characteristics of the 14 patients are presented in Table I.

Complete remission was achieved in all patients with nonmetastatic and low-risk metastatic diseases and in 4 (57\%) of the 7 patients with highrisk metastatic disease. However, 3 patients died.

The surgical procedures and their indications are listed in Table II. Total abdominal hysterectomy was performed in 14 patients.

All hysterectomies were carried out transabdominally. No operative mortality or morbidity occurred. Five (35.7\%) patients had post-operative chemotherapy after hysterectomy since they had pulmonary metastasis. Four (28.6\%) patients received neither preoperative chemotherapy nor postoperative chemotherapy.

Average follow-up duration after hysterectomy was $92.6 \pm 38.5$ months (range: $39-180$ months). There was no disease recurrence or mortality during this period. Pathological diagnosis of the patients who had hysterectomy was choriocarcinoma in 9 (64.3\%) cases, invasive mole in 3 (21.5\%) cases, and placental site tumor in 2 (14.2\%) cases (Table III).

\section{Discussion}

The first treatment to apply in trophoblastic diseases is evacuation of the uterus with vacuum curettage. Then, the patients are put on $\beta$-hCG follow-ups. In the patients with high $\beta$-hCG follow-ups, vacuum curettage can be applied several times. In this way, high cure rates and fertility potential can be achieved. If $\beta$-hCG levels do not drop, chemotherapy is applied. Gestational tro- 
Table I. Patient characteristics $(n=14)$

\begin{tabular}{|lc|}
\hline Characteristics & Results \\
\hline Antecedent pregnancy: & $10(71.4 \%)$ \\
\hline Hydatidiform mole & $3(21.4 \%)$ \\
\hline Abortion & $1(7.1 \%)$ \\
\hline Full-term & $10(71.4 \%)$ \\
\hline Preoperative chemotherapy: & $4(28.5 \%)$ \\
\hline Yes & \\
\hline No & $5(57.1 \%)$ \\
\hline Postoperative chemotherapy: & $9(64.2 \%)$ \\
\hline Yes & $9(14.2 \%)$ \\
\hline No & $4(50 \%)$ \\
\hline No. of preoperative chemotherapy regimens: \\
\hline 1
\end{tabular}

phoblastic tumors are chemosensitive. They generally respond well to chemotherapy. Resistance to chemotherapy is seen in a small group of the patients [3-5]. In this group, total abdominal hysterectomy needs to be performed. Hysterectomy can also be performed in situations other than chemotherapy resistance, especially in cases such as tumor perforation, incessant bleeding, infection and intraabdominal septicemia. Especially, in patients of advanced age, hysterectomy can be considered as a treatment option [6, 7]. Hysterectomies were performed in 14 patients diagnosed with GTN at the study center during a period of 15 years. Pisal et al. reported the rate of hysterectomy for GTN as 1/150 [8]. In patients whose disease is limited to the uterus, patients who are above 40 years of age and patients who have completed their fertility, hysterectomy is the best convenient procedure to lower the risk of metastatic or invasive disease or to minimize the chemotherapy dosage. Hysterectomy combined with chemotherapy is also a therapeutic option for the patients in whom bleeding cannot be stopped by
Table II. Hysterectomy indications in the patients with gestational trophoblastic neoplasms

\begin{tabular}{|lcc|}
\hline Indications & N & $\%$ \\
\hline Resistance to chemotherapy & 7 & 50 \\
\hline Treatment preference & 4 & 28.5 \\
\hline Bleeding & 3 & 21.5 \\
\hline Total & 14 & 100 \\
\hline
\end{tabular}

Table III. Pathological diagnosis of the patients who had hysterectomy

\begin{tabular}{|lcc|}
\hline Pathologic diagnosis & N & $\%$ \\
\hline Choriocarcinoma & 9 & 64.3 \\
\hline Invasive mole & 3 & 21.5 \\
\hline Placental site tumor & 2 & 14.2 \\
\hline Total & 14 & 100 \\
\hline
\end{tabular}

vascular embolization. Vascular embolization can be effectively used in young patients who want to conceive [9-11]. In this study, the patients who underwent hysterectomy had an average age of 48 and all of them completed their fertility, and therefore embolization was not performed.

Although surgery is a safe and effective method for the treatment of GTNs, it should not be considered as the first treatment choice. During the surgery, manipulation of the uterus might hematogenously spread the tumor. For this reason, some surgeons suggest that prophylactic chemotherapy should be administered before surgery. Ten $(71.4 \%)$ of our patients received prophylactic chemotherapy. Another reason is that vascularity in the pelvic area has increased in gestational trophoblastic disease. Arteriovenous malformations have increased. For this reason, damage that can occur in the pelvic area veins during the surgery may lead to complications such as serious bleeding and hematoma. Especially in invasive diseases when the tumor comes out of the uterus, problems such as hemostasis, major hemorrhage and adjacent organ injuries may occur. Apart from these issues, the general complications of hysterectomy rule out this method as the first treatment option [12-14].

In our series, no complications occurred during or after the hysterectomy. No chemotherapy was administered in the pre-operative period in order to prevent the tumor from spreading. In 5 (35.7\%) of our patients chemotherapy regimens were continued in the post-operative period because of the pulmonary metastasis. Four (28.6\%) patients never had chemotherapy in the pre-operative and post-operative periods. In these patients, hysterectomy was sufficient. 
In conclusion, hysterectomy appears to be a safe and effective method, especially in the treatment of patients with gestational trophoblastic disease who have advanced age and an unfavorable response to chemotherapy.

\section{Conflict of interest}

The authors declare no conflict of interest.

\section{References}

1. Brewer JI, Eckman TR, Dolkart RE, Torok EE, Webster A. Gestational trophoblastic disease. A comparative study of the results of therapy in patients with invasive mole and with choriocarcinoma. Am J Obstet Gynecol 1971; 109: 335-40.

2. Jelincic D, Hudelist G, Singer CF, et al. Clinicopathologic profile of gestational trophoblastic disease. Wien Klin Wochenschr 2003; 115: 29-35.

3. Gershenson DM, McGuire PW, Gore M, Quinn MA, Thomas G. Gynecologic cancer: controversies in management. 9th ed. Elsevier Churchill Livingstone USA, 2004; 555-70.

4. Tscherne G. [New aspects of diagnosis, treatment and follow-up of trophoblastic tumours]. Wien Klin Wochenschr Suppl 1979; 103: 1-21.

5. Aoki Y, Kodama S, Kurata H, Kase H, Tanaka K. Failure of high-dose chemotherapy with peripheral blood stem cell support for refractory placental site trophoblastic tumor. Gynecol Obstet Invest 1999; 47: 214-6.

6. Hammond CB, Weed JC Jr, Currie JL. The role of operation in the current therapy of gestational trophoblastic disease. Am J Obstet Gynecol 1980; 136: 844-58.

7. Jones WB, Romain K, Erlandson RA, Burt ME, Lewis JL Jr. Thoracotomy in the management of gestational choriocarcinoma. A clinicopathologic study. Cancer 1993; 72: 2175-81.

8. Pisal N, North C, Tidy J, Hancock B. Role of hysterectomy in management of gestational trophoblastic disease. Gynecol Oncol 2002; 87: 190-2.

9. Soper JT. Surgical therapy for gestational trophoblastic disease. J Reprod Med 1994; 39: 168-74.

10. Sand PK, Lurain JR, Brewer JI. Repeat gestational trophoblastic disease. Obstet Gynecol 1984; 63: 140-4.

11. Suzuka K, Matsui H, litsuka Y, Yamazawa K, Seki K, Sekiya S. Adjuvant hysterectomy in low-risk gestational trophoblastic disease. Obstet Gynecol 2001; 97: 431-4.

12. Haas JF. Pregnancy in association with a newly diagnosed cancer: a population-based epidemiologic assessment. Int J Cancer 1984; 34: 229-35.

13. Tse KY, Chan KK, Tam KF, Ngan HY. 20-year experience of managing profuse bleeding in gestational trophoblastic disease. J Reprod Med 2007; 52: 397-401.

14. Ohmaru T, Yamakawa H, Netsu S, Nokubi M, Konno R. Placental site trophoblastic tumor (PSTT) with multiple metastases and extremely poor prognosis. Int I Clin Oncol 2009; 14: 452-6. 